PO002

\title{
COLORIMETRIC VALUES OF IMAGE SKIN COLOR IN THE WHOLE FACE AND CHEEK PART, AND THEIR RELATION TO SUBJECTIVE EVALUATION
}

Miyoshi Ayama et al.

DOI 10.25039/x46.2019.PO002

from

CIE x046:2019

Proceedings

of the

29th CIE SESSION

Washington D.C., USA, June 14 - 22, 2019

(DOI 10.25039/x46.2019)

The paper has been presented at the 29th CIE Session, Washington D.C., USA, June 14-22, 2019. It has not been peer-reviewed by CIE.

(C) CIE 2019

All rights reserved. Unless otherwise specified, no part of this publication may be reproduced or utilized in any form or by any means, electronic or mechanical, including photocopying and microfilm, without permission in writing from CIE Central Bureau at the address below. Any mention of organizations or products does not imply endorsement by the CIE.

This paper is made available open access for individual use. However, in all other cases all rights are reserved unless explicit permission is sought from and given by the CIE.

CIE Central Bureau

Babenbergerstrasse 9

A-1010 Vienna

Austria

Tel.: +4317143187

e-mail: ciecb@cie.co.at

www.cie.co.at 


\title{
COLORIMETRIC VALUES OF IMAGE SKIN COLOR IN THE WHOLE FACE AND CHEEK PART, AND THEIR RELATION TO SUBJECTIVE EVALUATION
}

\author{
Ayama, M. ${ }^{1,2}$, Shiromizu, S. ${ }^{1}$, Kawame, K. ${ }^{1}$, Arimoto, $\mathrm{K}^{3}{ }^{3}$, Kimura, M. ${ }^{3}$, Hata, $\mathrm{H}^{3}{ }^{3}$, Koshino, M. ${ }^{3}$, \\ Ishikawa, T. ${ }^{1}$ \\ ${ }^{1}$ School of Engineering, Utsunomiya University, JAPAN \\ ${ }^{2}$ Center for Optical Research \& Education, Utsunomiya University, JAPAN \\ ${ }^{3}$ Shiseido Global Innovation Center, JAPAN \\ miyoshi@is.utsunomiya-u.ac.jp
}

DOI 10.25039/x46.2019.PO002

\begin{abstract}
Recently, skin color of facial image on a display becomes as important as that of real face. A number of applications have been used to retouch original face picture. To investigate the area in a color-space where the rating score of "Finish-up impression", which is one of the important evaluation points for Japanese women, exceeds an acceptable level, subjective evaluation experiment was carried out using a number of test stimuli in a wide range of color difference from the original face image. In addition to that, other subjective evaluations such as "Brightness or darkness" or "Redness or yellowness" were done. Colorimetric values of the small part in the cheek and the whole face were measured and compared with each other.
\end{abstract}

Keywords: Skin color, Facial image, Finish-up impression, Subjective evaluation

\section{Introduction}

Recently, importance of skin color of facial image on a display is being increased in variety of scenes, such as portraits in application forms, pictures in photo sharing sites, etc. To improve the impression of face on a display, a number of applications have been proposed and used to retouch original face picture. A number of studies have been done to reveal the relation between skin color of facial image and attractiveness, perceived whiteness, preference, naturalness and/or other attributes, based on subjective evaluation [1-9]. Some studies employed large number of different faces from database of face image $[1,2,6,7]$, while other studies used test stimuli rendered in various ways from relatively small number of original faces $[3-5,8]$. Considering the application to make-up cosmetics, the latter approach, i.e., using a large number of color rendered images of the same face is more useful than the former approach. Samson et al. [3] used the image of one senior British woman's face and created test stimuli by smoothing color distribution/surface topography, and did the rating experiment for perceived age and health. Results indicated that the smooth surface gives young looking, while color uniformity provides healthy impression. Yoshikawa et al. [4] created various color-rendered facial images of the same artificial face as test stimuli and did the whiteness matching using a series of scale-images of which lightness is changed in the same step. Results showed that decreasing chroma increases perceived whiteness. Zeng et al. [5] used 12 face images, the skin color of them was morphed toward various color-center, and carried out the preference evaluation experiment. Color centers close to $\left(a^{*}, b^{*}\right)=(20,20)$ were the most preferable for all ethnic facial images. Wang et al. [8] used 4 different faces, each of them was rendered into 26 images of different color surrounding the original one, and carried out the subjective evaluation experiments using five attributes such as "Likable" or "Health". In above studies, number of color-rendered images from an original face is relatively small up to about 50 , and thus the change of subjective rating score in color space is not shown.

In the case of evaluating a make-up face, finish-up impression is quite important for Japanese women. It is to evaluate the face on the assumption that the model made-up her face, and no study has been performed focusing on the issue. Therefore, our primary aim is to investigate the area in a color-space where the rating score of "Finish-up impression" exceeds an acceptable level. In addition to that, we aim to clarify the relations between "Finish-up impression" and other subjective evaluations such as "Brightness or darkness" or "Redness or yellowness". 
To identify the representative skin color of facial image is another important issue [9]. In the present study, colorimetric values of the small part in the cheek and the whole face in face image were compared. Color in a small part in the cheek was often employed as the complexion color in previous studies using real models [10]. Although the spectral components of real face and display are different, such comparison would be still useful to make rough estimation of chromaticities and lightness of whole face from colorimetric values of limited portion in the cheek.

\section{Experiment}

\subsection{Test Stimuli}

A facial image of 3 Japanese women in her twenties without makeup was taken by a digital camera and set as an original image. We call them test faces $A, B$, and $C$, in the present study. Picture of the test face $A$ is indicated in Figure 1. In the evaluation experiments, a black bar was not presented. For each of the test faces, image group composed of 343 was created in which the $L^{*}, a^{*}$, and $b^{*}$ of each pixel in the skin color area were changed with nearly a constant step of $\Delta L^{*}=2, \Delta a^{*}=2$, and $\Delta b^{*}$ $=2$, from -6 to 6 along 3 axes.

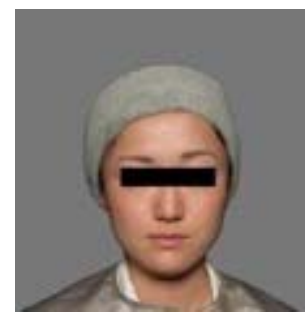

\subsection{Colorimetry}

Figure 1 - Example of test image

For all the test stimuli, skin color part was extracted by deleting the eyes, eyebrows, and lips as shown in Figure 2. Chromaticity and luminance of the small areas in the left and right cheeks of the test images on the display were measured using spectroradiometer (Konica Minolta CS2000). Average values of the left and right cheeks of 119 test images are plotted in Figure 3 with blue diamonds. The horizontal axes in the left and right figures are $a^{*}$ and $b^{*}$, respectively, and the vertical axis is the metric lightness $L^{*}$.

For all the test stimuli, chromaticities and luminance of the same skin-part-extracted images on the display as described above were also measured using 2D colorimeter (Konica Minolta CA2500). $L^{*} a^{*} b^{*}$ values of each pixel are calculated employing the maximum white of the display as the reference white. In the 2D colorimetry, which colorimetric value, an average of the mean, mode, or other statistical value of the whole face is the most appropriate to represent the face color is an issue to be studied. In this study, we employed the average colorimetric values of the top $60 \%$ in the appearance-frequency of the whole-face image as a representative color [9]. The values were calculated for each of 119 test images selected from 343 test images, and plotted in Figure 3 with red circles. For all test images measured here, the $L^{*}$ is lower than the cheek color about the value of $10, b^{*}$ is larger than about the value of 2 to 3 , while $a^{*}$ value does not show systematic shift. Results are summarized that the color of small area in the cheek is lighter, whiter, and reddish than the whole face.

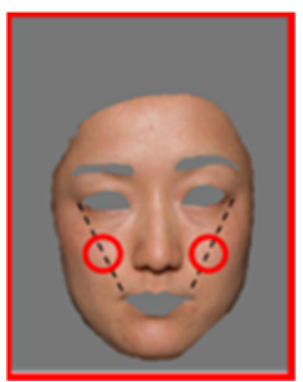

Figure 2 - Area of colorimetric measurements for the cheeks

(circles) and the whole face (square). Test face A.

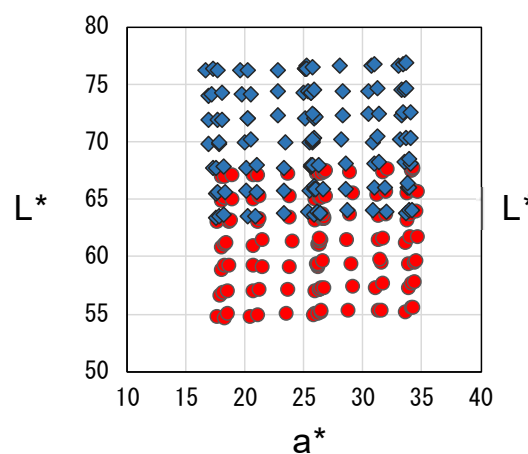

Whole face

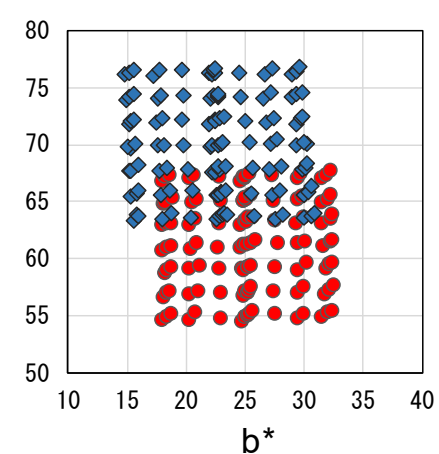

Cheek color
Figure 3 - Comparison between colorimetric values of the cheeks (blue diamonds) and the whole face (red circles). The left and right figures are plotted in the $a^{*}-L^{*}$ plane and $b^{*}$ - $L^{*}$ plane, respectively, for images of the test face $A$. 


\subsection{Subjective Evaluation}

We employed the following words for subjective evaluation, "Finish-up impression", "Color affinity to her skin", "Difference between face and neck color", "Brightness or darkness", and "Redness or yellowness". For each of the words, subjective evaluation using the rating scores from 1 to 5 were done. In the present study, results of "Finish-up impression", "Brightness or darkness", and "Redness or yellowness" are shown. In the case of "Finish-up impression", scores of 5, 3, and 1 correspond to "like", "neutral", and "dislike" as a finish-up of make-up face, respectively. In the case of "Brightness or darkness", scores of 5, 3, and 1 correspond to perceptually "very bright", "neutral", and "very dark", respectively. In the case of "Redness or yellowness", scores of 5,3 , and 1 correspond to "strong tinge of yellow", "neutral", and "strong tinge of red", respectively.

\subsection{Experimental procedure}

In the experiment, a test image was presented on a display (EIZO ColorEdge CG277), and the observer was asked to do subjective evaluations described above, and respond orally to the experimenter. Order of evaluation words was randomized between sessions and observers. Test image presentation was also randomized. Uniform gray image $(R, G$, and $B=119)$ was appeared between test image presentations. In one session, 114 or 115 images were presented. Three sessions to cover the whole test images were regarded as 1 set. Three sets of different presentation orders were carried out for each observer.

\subsection{Observers}

Five male students and five female students aged 21 to 23 , with normal acuity and color vision participated the experiment.

\section{Results}

Contour maps of the rating score of "Finish-up impression" and "Bright vs Dark" in the $a^{*}-b^{*}$ plane at $\Delta L^{*}=0,2,4$, and 6 for test face $A$ based on the average scores of 10 observers are indicated in Figures 4 and 5 , respectively. $\Delta L^{*}$ values are nominal and fluctuated among the test images of the same nominal $\Delta L^{*}$ within the range of 0.8 . Hereafter, colorimetric values based on the measurement of whole face images (2D colorimetry), are employed. Those are the average values of top $60 \%$ in the appearance-frequency of the whole-face image [9].

In the results of "Finish up Impression", average scores are entirely lower than 3.0 in the planes where $\Delta L^{*}<0$, and thus those planes are not shown. As shown in Figure 4 , acceptable area (rating score $>3.4$, for convenience) is very small in the $\Delta L^{*}=0$ plane at $\left(a^{*}, b^{*}\right)=(27,27)$. The size of acceptable area increases with $\Delta L^{*}$ and shows the maximum size and highest score in the $\Delta L^{*}=4$ plane around at $\left(a^{*}, b^{*}\right)=(25,25)$. The acceptable area size shrinks slightly in the $\Delta L^{*}=6$ plane, indicating that not necessarily the lighter the better. The acceptable area in the $\Delta L^{*}=4$ plane is near the original face color but elongates along the hue angle $H$ of about $45 \mathrm{deg}$ which is slightly yellowish than the original face. Metric chroma of acceptable area varies from about 34 to 38 , while that of original is 36.5 , indicating that not necessarily the whiter the better.

Results of "Bright vs Dark" evaluation shown in Figure 5 indicate interesting tendency. In all planes from $\Delta L^{*}=0$ to 6 , high score area is in the smaller values of $a^{*}$ and $b^{*}$, i.e. the smaller $C^{*}$ in the same $\Delta L^{*}$ plane, the brighter perception, which is opposite to the Helmholtz-Kohlrausch effect [12]. Shape of contour lines are quite different from those in Figure 4. Comparison between Figure 4 and 5, the rating score of "Bright vs Dark" evaluation in the acceptable area of "Finish up Impression" is around 3.4 to 4.2. Brighter than the original image is needed, but not too bright is important for better "Finish-up Impression".

Contour maps of the rating score of "Redness or Yellowness" in the $\mathrm{a}^{*}-\mathrm{b}^{*}$ plane at $\Delta L^{*}=-6,0$, and 6 , for observer $A$ based on the average scores of 10 observers are indicated in Figures 6. Contour lines indicate the change of apparent hue from reddish to yellowish in the chromaticity range of test stimuli. It is worth noting that the change is more rapid in the $\Delta L^{*}=-6$ plane than that in the $\Delta L^{*}=0$ and $\Delta L^{*}=6$ planes. Neutral zone, score of around 3.0, narrows as the decrease of $\Delta L^{*}$. This indicates that the lower the $\Delta L^{*}$, the more sensitive to the change of hue. 

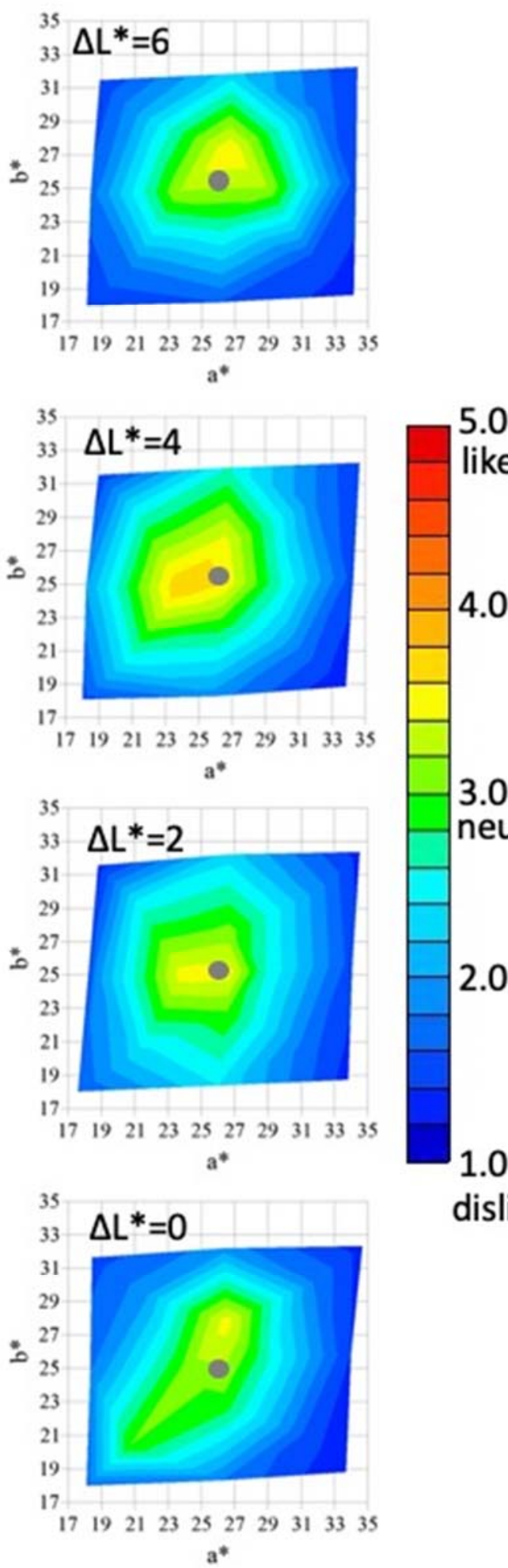

Figure 4 - Contour maps of the rating scores for "Finish-up impression" in the $a^{*}-b^{*}$ plane for images of the test face A. Gray circle denote the $\left(a^{*}, b^{*}\right)$ of the original test image.

3.0

2.0

1.0
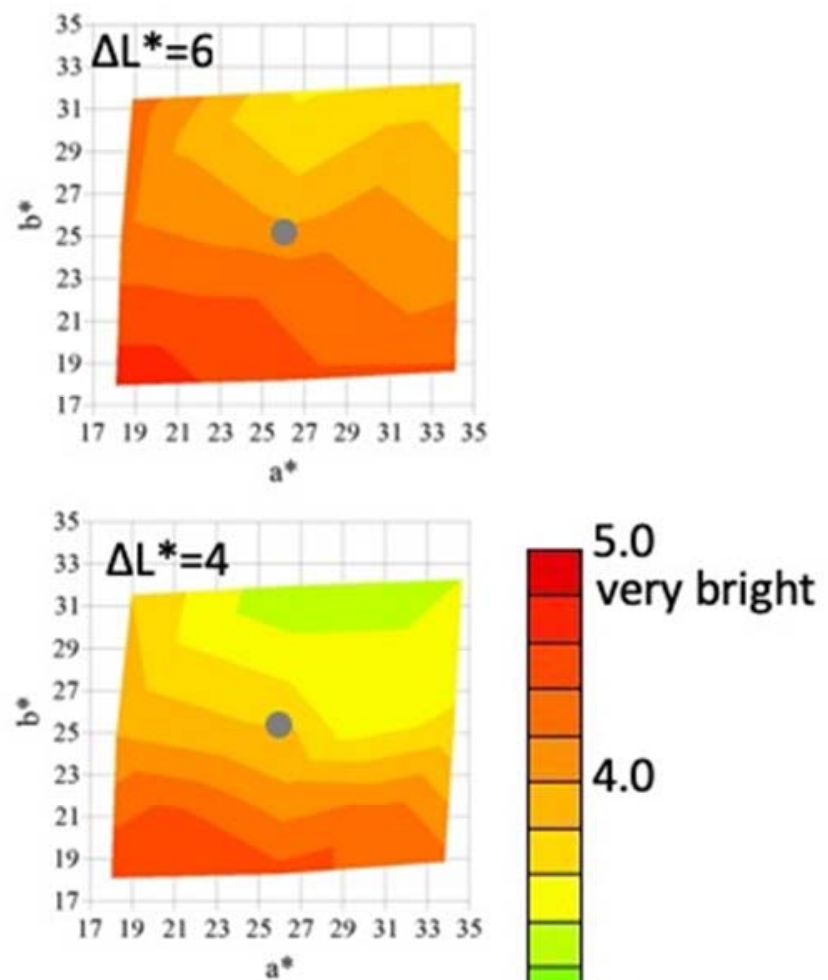
neutral

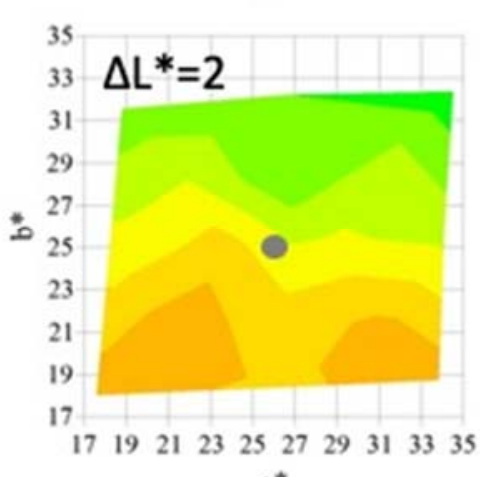

3.0

neutral

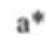

1.0

dislike

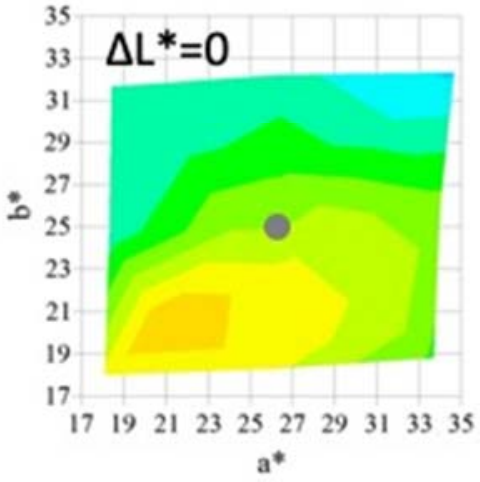

very dark

Figure 5 - Contour maps of the rating scores for "Bright vs Dark" in the a*$b^{*}$ plane for images of the test face $A$. Gray circle denote the $\left(a^{*}, b^{*}\right)$ of the original test image. 


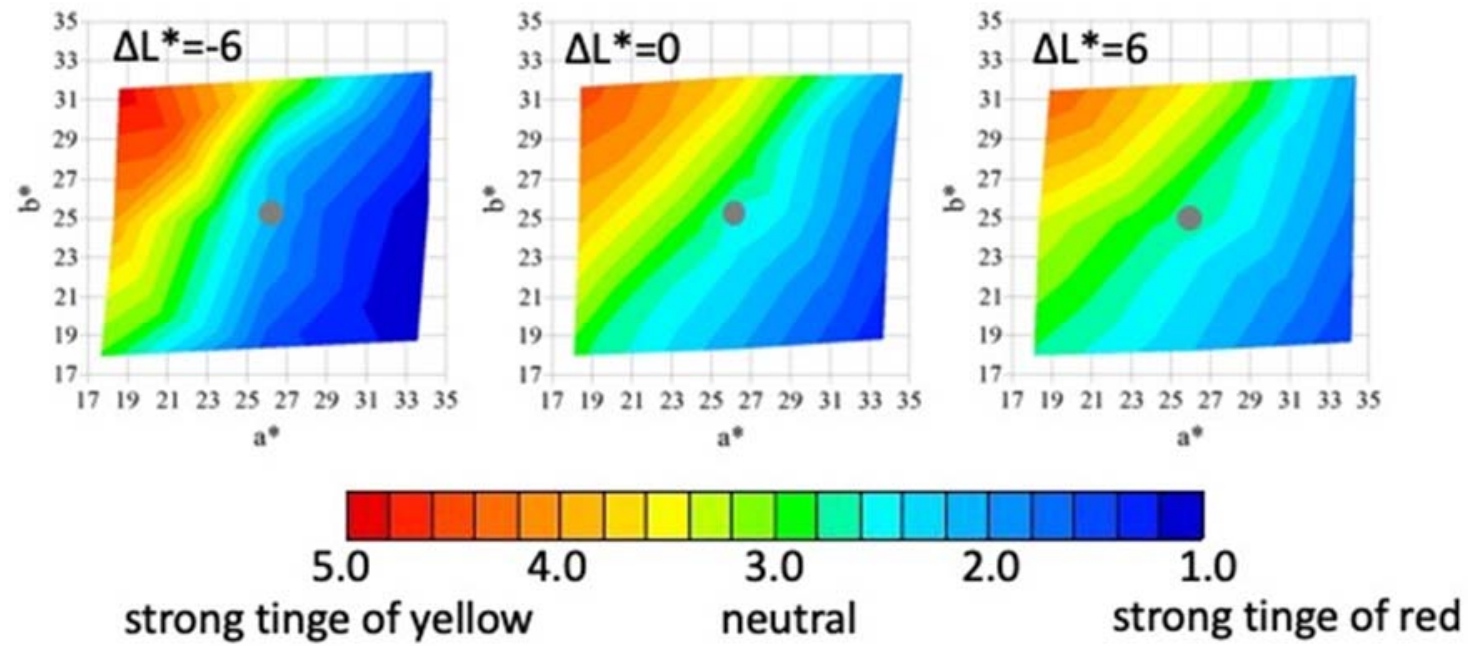

Figure 6 - Contour maps of the rating scores for "Yellowish vs Reddish" in the $a^{*}-b^{*}$
plane for images of the test face A. Gray circle denote the $\left(a^{*}, b^{*}\right)$ of the original test
image.

\section{Conclusion}

Colorimetric values of whole face and small parts in the cheek were compared in a large number of facial image. Metric lightness $L^{*}$ of the cheek is higher, $a^{*}$ stays nearly the same, and $b^{*}$ is smaller. In other words, the cheek is lighter, whiter, and reddish than the whole face. and reddish than the whole face. Similar tendency was observed for other 2 test faces.

Measurements using a large number of test images revealed how the rating score of subjective evaluation changes in the CIELAB space, and figured out the area of acceptable rating scores. From overall results of "Finish-up impression", "Brightness or Darkness", and "Redness or Yellowness", good impression for the make-up face is obtained in the area in a color-space where brightness is slightly higher than, saturation is not away from, and hue is less reddish than the original one. Our study shows that neither 'the brighter the better', nor 'the whiter the more preferable'. "Finish-up impression" is close to attractiveness or preference, but our results showed somehow different. Relations to other evaluations such as "Color affinity to her skin" and "Difference between face and neck color" are to be investigated.

\section{References}

1. Fink, B. et al. 2001. Human (Homo sapiens) Facial Attractiveness in Relation to Skin Texture and Color. J. Comp. Psychol., 115, 92-99.

2. Fink, B., Matts, P. J. 2008. The Effects of Skin Colour Distribution and Topography Cues on the Perception of Female Facial Age and Health. J. Eur. Acad. Darmatol. Venereol., 22, 493-498.

3. Samson, N. et al. 2011. Interaction of Skin Color Distribution and Skin Surface Topography Cues in the Perception of Female Facial Age and Health. J. Cosmet. Dermato., 10, 78-84.

4. Yoshikawa, H. et al. 2012. Effect of Chromatic Components on Facial Skin Whiteness. Color Res. Appl., 37, 281-291.

5. Zeng, H. Luo, R. 2013. Colour and Tolerance of Preferred Skin Colours on Digital Photographic Images. Color Res. Appl., 38, 30-45.

6. Zhao, B. et al. 2017.Developing a Model for Predicting Whiteness of Human Face. Proceedings of the $13^{\text {th }}$ AIC Congress, USB.

7. Zhao, B. et al. 2017. Investigation of Facial Attractiveness Using a Facial Image Database. 
Proceedings of the $13^{\text {th }}$ AIC Congress 2017, USB.

8. Wang, M. et al., 2017.The Impact of Skin Colour on Facial Impressions. Proceedings of the $13^{\text {th }}$ AIC Congress, USB.

9. Kawame, et al. 2019. Representative Color of the Whole-face and its Relation to Finish-up Impression. Proceedings of CIE2019.

10. Yano, T., Hashimoto, K. 1997. Preference for Japanese Complexion Color under Illumination. Color Res. Appl., 22, 269-274.

11. Shiromizu, S. et al. 2018. KANSEI Evaluation of Skin Color of Young Japanese Women on a Display and the Relation to Colorimetric Values of Whole Face. Proceedings of IDW2018, 960-963.

12. Ayama, M. Ikeda, M. 1998. Brightness-to-Luminance Ratio of Colored Light in the Entire Chromaticity Diagram. Color Res. Appl., 23, 274-287. 